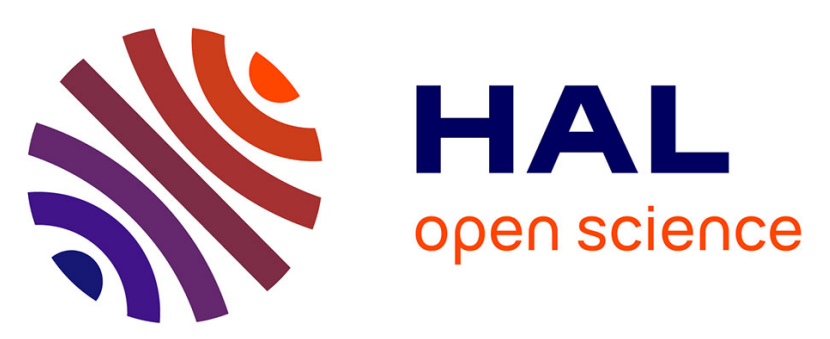

\title{
A scaffold of accessory subunits links the peripheral arm and the distal proton pumping module of mitochondrial complex I
}

Heike Angerer, Klaus Zwicker, Zibiernisha Wumaier, Lucie Sokolova, Heinrich Heide, Mirco Steger, Silke Kaiser, Esther Nübel, Bernhard Brutschy, Michael Radermacher, et al.

\section{To cite this version:}

Heike Angerer, Klaus Zwicker, Zibiernisha Wumaier, Lucie Sokolova, Heinrich Heide, et al.. A scaffold of accessory subunits links the peripheral arm and the distal proton pumping module of mitochondrial complex I. Biochemical Journal, 2011, 437 (2), pp.279-288. 10.1042/BJ20110359 . hal-00605259

\section{HAL Id: hal-00605259 \\ https://hal.science/hal-00605259}

Submitted on 1 Jul 2011

HAL is a multi-disciplinary open access archive for the deposit and dissemination of scientific research documents, whether they are published or not. The documents may come from teaching and research institutions in France or abroad, or from public or private research centers.
L'archive ouverte pluridisciplinaire HAL, est destinée au dépôt et à la diffusion de documents scientifiques de niveau recherche, publiés ou non, émanant des établissements d'enseignement et de recherche français ou étrangers, des laboratoires publics ou privés. 


\section{A scaffold of accessory subunits links the peripheral arm and the distal proton pumping module of mitochondrial complex I}

Heike ANGERER*, Klaus ZWICKER*, Zibiernisha WUMAIER*, Lucie SOKOLOVA ${ }^{\dagger}$, Heinrich HEIDE*, Mirco STEGER*, Silke KAISER*, Esther NÜBEL*, Bernhard BRUTSCHY ${ }^{\dagger}$, Michael RADERMACHER ${ }^{\$}$, Ulrich BRANDT*, Volker ZICKERMANN*1

\footnotetext{
* Molecular Bioenergetics Group, Medical School, Cluster of Excellence Frankfurt "Macromolecular Complexes", Center for Membrane Proteomics, Goethe-University, D-60590 Frankfurt am Main, Germany

${ }^{\dagger}$ Institute of Physical and Theoretical Chemistry, Cluster of Excellence Frankfurt "Macromolecular Complexes", Center for Membrane Proteomics, Goethe University, D-60439 Frankfurt am Main, Germany

${ }^{\$}$ University of Vermont, College of Medicine, Department of Molecular Physiology and Biophysics, Burlington, Vermont 05405, USA

${ }^{1}$ Correspondence may be addressed to V. Zickermann (email:Zickermann@zbc.kgu.de)
}

Short title: Topology of complex I

\section{Synopsis}

Mitochondrial NADH:ubiquinone oxidoreductase (complex I) is a very large membrane protein complex with a central function in energy metabolism. Complex I from the aerobic yeast Yarrowia lipolytica comprises 14 central subunits that harbour the bioenergetic core functions and at least 28 accessory subunits. Despite progress in structure determination the position of individual accessory subunits in the enzyme complex remains largely unknown.

Proteomic analysis of subcomplex I $\delta$ revealed that it lacked eleven subunits including the central subunits ND1 and ND3 forming the interface between the peripheral and the membrane arm in bacterial complex I. This unexpected observation provided insight into the structural organization of the connection between the two major parts of mitochondrial complex I. Combining recent structural information, biochemical evidence on the assignment of individual subunits to the subdomains of complex I and sequence based predictions for the targeting of subunits to different mitochondrial compartments, we derived a model for the arrangement of the subunits in the membrane arm of mitochondrial complex I.

Keywords: membrane protein, mitochondria, NADH dehydrogenase, protein import, respiratory chain, subcomplex

Abbreviations: ESI-MS, electrospray ionisation- mass spectrometry; FMN, flavin-mononucleotide; LILBID-MS, laser induced liquid bead ion desorption mass spectrometry; HAR, hexaammineruthenium(III)-chloride; DBQ, decylubiquinone; DDM, $\beta$-D-dodecyl-maltoside, DQA, 2decyl-4-quinazolinylamine; LDAO, lauryl dimethylamine oxide; $\mathrm{Q}_{1}$, ubiquinone-1; STMD, single transmembrane domain 


\section{INTRODUCTION}

Proton pumping NADH:ubiquinone oxidoreductase (complex I, EC 1.6.5.3) is the largest membrane protein complex in the respiratory chain of many bacteria and mitochondria [1-3]. Coupling the twoelectron transfer reaction from $\mathrm{NADH}$ to ubiquinone to the vectorial translocation of four protons, mitochondrial complex I provides a major portion of the proton motive force that drives ATP synthesis. The enzyme is a source of reactive oxygen species and has been implicated in the pathogenesis of a number of human neurodegenerative diseases [4].

Mitochondrial complex I is composed of more than 40 different subunits subdivided into central and accessory subunits. The fourteen central subunits carry out the bioenergetic function of complex I and are conserved throughout species, from bacteria to mammals. They can be assigned to three functional modules [3] that harbour the bioenergetic core functions NADH oxidation ( $\mathrm{N}$-module), ubiquinone reduction (Q-module) and proton translocation (P-module). The substrate binding sites are connected by a chain of iron-sulfur clusters. The function of the accessory subunits of mitochondrial complex I is largely unknown. Individual subunits were suggested to play a role in assembly, regulation, and stabilization of the complex [3;5-7]. In complex I from the strictly aerobic yeast Yarrowia lipolytica so far 28 accessory subunits with a total mass of $421.0 \mathrm{kDa}$ were identified corresponding to $43.7 \%$ of the total mass of $963.7 \mathrm{kDa}[8]$ (Nübel et al., in preparation).

Electron microscopy revealed that complex I from mammalian and fungal mitochondria like that from eubacteria is L-shaped with a hydrophilic peripheral arm and a hydrophobic membrane arm [9]. Recently, X-ray crystallographic analysis provided insight into the overall architecture of prokaryotic [10] and eukaryotic [11] complex I at intermediate resolution. The X-ray structure of a bacterial peripheral arm fragment had been solved earlier at up to $3.1 \AA$ resolution [12]. It comprises the N- and the Q-module that together perform all the redox-chemistry. As had been suggested before [13;14], the terminal iron-sulfur cluster $\mathrm{N} 2$ that is the immediate electron donor for ubiquinone, was found at a remarkable distance from the membrane domain [10;11]. The membrane arm or P-module is subdivided into a proximal $\left(\mathrm{P}_{\mathrm{P}}\right)$ and a distal $\left(\mathrm{P}_{\mathrm{D}}\right)$ domain [11]. Several lines of evidence suggest that both parts of the P-module contribute to proton translocation. An elongated presumably $\alpha$-helical element that is laterally associated with the membrane arm was discovered that connects the proximal and distal domains of the P-module and has been suggested to play a key role in energy transmission within the membrane arm [10;11]. Overall, the relative arrangement of functional modules in complex I indicates a spatial separation of redox chemistry and proton translocation essentially excluding direct mechanisms of energy transfer. It rather suggests that complex I operates by a conformational coupling mechanism.

Complex I from many bacteria is too unstable to be prepared in intact form. This has only been achieved with complexes from Escherichia coli [15], Paracoccus denitrificans [16] and some thermophilic eubacteria [10;17]. Complex I purified from these species still tends to disintegrate very easily into the peripheral arm comprising the Q- and N-modules and the membrane integral P-module. Also the compositions of several subcomplexes that have been prepared biochemically from bovine complex I by applying mild chaotropic conditions [18-21] reflect the modular architecture of complex $\mathrm{I}$, although there are some notable discrepancies. Subcomplex I $\alpha$ comprises the Q- and N-module of the peripheral arm and a sector of the $\mathrm{P}_{\mathrm{P}}$-module of the membrane arm while subcomplex $\mathrm{I} \beta$ essentially corresponds to the distal membrane arm module $\mathrm{P}_{\mathrm{D}}$. Further disassembly of subcomplex $\mathrm{I} \alpha$ leads to formation of subcomplex I $\lambda$ that is more hydrophilic but still contains two accessory subunits with predicted transmembrane helices.

A subcomplex similar to bovine subcomplex $I \lambda$ has been generated previously by disintegration of complex I from the strictly aerobic yeast Y. lipolytica [22]. Here we extended these studies and prepared a new type of subcomplex still comprising the Q-, N- and $\mathrm{P}_{\mathrm{D}}$-module of complex $\mathrm{I}$, but lacking a significant portion of the $\mathrm{P}_{\mathrm{P}}$-module. Our results suggest that a rather stable connection must exist in mitochondrial complex I between the peripheral arm and the distal membrane arm domain that involves specific accessory subunits. Combining available structural information, biochemical data, 
and sequence analysis, we have derived a topological model for the arrangement of the central and accessory subunits in the membrane arm of complex I.

\section{EXPERIMENTAL}

\section{Preparation of subcomplex I $\delta$ from $Y$. lipolytica, catalytic activity and EPR spectroscopy}

Complex I from Y. lipolytica strain PIPO (His-tagged 30-kDa subunit) was prepared as described [23]. A strain variant of PIPO expressing subunit NUJM-Strep-tagII (His-tagged 30-kDa subunit and StrepII-tagged NUJM) was generated as described elsewhere (Nübel et al., in preparation). Subcomplex I $\delta$ was prepared by incubation of $2 \mathrm{mg}$ purified complex I in $5 \mathrm{~mL}$ incubation buffer (20 $\mathrm{mM} \mathrm{NaP}, 400 \mathrm{mM} \mathrm{NaCl}, 20 \mathrm{mM}$ imidazole, $1 \mathrm{mM} \mathrm{MgCl}$, $1 \% \mathrm{LDAO}, \mathrm{pH}$ 7.2) on ice for $2 \mathrm{~h}$. The sample was loaded on a Ni-NTA column and washed with 12 column volumes LDAO-washing buffer $(20 \mathrm{mM} \mathrm{NaP}$, $400 \mathrm{mM} \mathrm{NaCl}, 20 \mathrm{mM}$ imidazole, $1 \%$ LDAO, pH 7.2). The detergent was exchanged to $\beta$-dodecylmaltoside (DDM) with 9 column volumes DDM-washing buffer $(20 \mathrm{mM} \mathrm{NaP}$ i $\mathrm{pH}, 400$ $\mathrm{mM} \mathrm{NaCl}, 20 \mathrm{mM}$ imidazole, $0.025 \%$ DDM, pH 7.2). Subcomplex I $\delta$ was eluted with 7 column volumes DDM-elution buffer $(20 \mathrm{mM} \mathrm{NaP}, 400 \mathrm{mM} \mathrm{NaCl}, 140 \mathrm{mM}$ imidazole, $0.025 \% \mathrm{DDM}, \mathrm{pH}$ 7.2). The concentrated sample (Amicon concentrator $50 \mathrm{kDa}$ cut off, Millipore) was applied to gel filtration (TSK $40007.8 \times 30)$ in gel filtration buffer ( $20 \mathrm{mM} \mathrm{Na}$-Mops, $100 \mathrm{mM} \mathrm{NaCl}, 1 \mathrm{mM}$ EDTA, $0.025 \%$ DDM, pH 7.2) and concentrated to $15 \mathrm{mg} / \mathrm{mL}$ final protein concentration. Protein concentration was determined according to a modified Lowry protocol using bovine serum albumin as standard protein.

NADH:HAR, NADH:DBQ, and NADH:Q ${ }_{1}$ oxidoreductase activity was measured essentially as described in [24]. X-band EPR spectra were obtained essentially as described in [25].

\section{Electrophoresis}

Blue-Native PAGE of $Y$. lipolytica mitochondria was performed using DDM (1 g/g protein) as detergent according to [26]. Molecular masses of complex I, complex V and complex III-dimer from Y. lipolytica mitochondrial membranes were assigned as described [27].

Subunits of protein complexes were separated by doubled SDS-PAGE (dSDS-PAGE) that was carried out as reported previously [28] with the minor change that protein samples were reduced using 130 mM DTT. Briefly, lanes from ID SDS-PAGE gels $(10 \%$ polyacrylamide, $6 \mathrm{M}$ urea $)$ were incubated in acidic solution containing $100 \mathrm{mM}$ Tris, $150 \mathrm{mM} \mathrm{HCl}, \mathrm{pH}<2$ for $30 \mathrm{~min}$ and analysed by second dimension SDS-PAGE using $16 \%$ polyacrylamide.

\section{ELISA and identification of subunit NUPM by western blot analysis and LC-MS/MS}

The standard ELISA using alkaline conditions for binding of complex I or subcomplex I $\delta$ to polystyrene microtiter plates were carried out as described [13]. Primary monoclonal antibodies against several subunits were used for subunit identification (anti-49-kDa and anti-PSST, anti-NUPM, two different anti-NESM antibodies and anti-NIAM) [13](Zickermann, unpublished).

We used a three-dimensional electrophoresis technique (BNE followed by dSDS-PAGE) to separate the protein subunits of complex I from $Y$. lipolytica and to characterize the monoclonal antibody 31A8. To prepare complex I for BN-PAGE, $0.3 \mathrm{~g} / \mathrm{g}$ DDM was added to one $\mathrm{mg}$ of chromatographically purified complex I [26]. The repurified complex I band was excised from the $\mathrm{BN}$-gel and the subunits were separated using dSDS-PAGE. Following electroblotting of the dSDSPAGE gels on polyvinylidenfluoride membranes [26], monoclonal antibody $31 \mathrm{~A} 8$ was used for 
immunological detection. A gel piece from a duplicate Commassie-stained gel was cut out for LCMS/MS analysis revealing binding of monoclonal antibody $31 \mathrm{~A} 8$ to subunit NUPM.

\section{Mass Spectrometry}

Laser Induced Liquid Bead Ion Desorption Mass Spectrometry (LILBID-MS) was done as described previously [29]. Protein bands were analyzed by Electrospray Ionization Mass Spectrometry (ESI-MS) after dSDS- or BN-PAGE. Gel spots were excised and in gel digested with trypsin as essentially described by Collins et al. [30]. In addition complete purified complex I was digested in solution (see Supplelmentary Table III). Briefly, about $70 \mu \mathrm{g}$ of complex I were reduced with DTT $(5 \mathrm{mM})$ and alkylated with iodoacetamide $(15 \mathrm{mM})$ in $50 \mathrm{mM}$ ammonium bicarbonate buffer and subsequently digested overnight with $1 \mu \mathrm{g}$ of trypsin or chymotrypsin in $0.01 \%$ ProteaseMAX ${ }^{\mathrm{TM}}$ surfactant $(\mathrm{w} / \mathrm{v})$ (Promega). The peptides were extracted from the digest using cation exchange. The dried peptides were redissolved in 5\% acetonitrile/ $0.5 \%$ formic acid for subsequent analysis by ESI-LC-MS/MS.

The extracted peptides were separated on a nano-HPLC column (PicoTip ${ }^{\mathrm{TM}}$, New Objective), packed with reversed phase silica (Hypersil Gold $\mathrm{C}_{18}, 3 \mu \mathrm{m}$, Thermo Scientific) and analysed by a LTQ Orbitrap XL mass spectrometer as described [31], except that gradients were adapted to the complexity of the sample.

\section{Electron microscopy}

Complex I decorated with monoclonal antibody anti-NUPM, 31A8, was analyzed by electron microscopy and image processing. The sample was first diluted to $0.48 \mathrm{mg} / \mathrm{ml}$ (buffer: $25 \mathrm{mM}$ $\mathrm{Na} /$ Mops pH 7.2, $100 \mathrm{mM} \mathrm{NaCl}, 0.025 \% \mathrm{DDM}$ ) and mixed with a two-fold molar excess of antibody. After dilution to $0.03 \mathrm{mg} / \mathrm{ml}$, the mixture was applied to carbon coated 400 Mesh copper grids and deep stain embedded with NanoW (Nanoprobes, Yaphank, NY) [32;33]. The micrographs were scanned on a SCAI flatbed scanner (Z/I Imaging Corporation, Huntsville, AL) with a calibrated pixel size of $4.02 \AA$ on the specimen scale. 755 single particle images were boxed out of the micrographs, using as only criterion that the particles showed the approximate size of complex I and were separated from their neighbor particles, without differentiation between labeled and unlabeled protein. The boxed particles were processed by centration followed by iterations of rotational translational alignments [34] correspondence analysis [35] classification [36], and multireference alignments, classification being the final step.

\section{Sequence analysis}

Prediction of N-terminal mitochondrial targeting sequences (MTS) was done using the MITOPROT algorithm [37]. Alignments were done with default parameters using the program ClustalW2[38]. For prediction of transmembrane segments we used the programs TMHMM and hmmtop [39;40].

\section{RESULTS}

\section{Subcomplex I $\delta$ lacks part of the proximal P-module}

Exposure of purified complex I to $1 \%$ of the zwitterionic detergent lauryl dimethylamine oxide (LDAO) reproducibly resulted in formation of a defined subcomplex as determined by Blue-Native (BN)-PAGE (Fig. 1A) and size exclusion chromatography (Fig. 1B). Expanding the established nomenclature of biochemically prepared subcomplexes $[18 ; 20]$ we termed the new subcomplex I $\delta$. We noted that in BN-PAGE the subcomplex migrated as a rather diffuse band and exhibited a pronounced tendency to form oligomers. The subunit composition of monomer and oligomers was found to be essentially identical (see below, Supplementary Table II). A total mass of $\sim 850 \mathrm{kDa}$ at peak maximum 
can be deduced from a series of prominent peaks in non-destructive laser induced liquid bead ion desorption mass spectrometry (LILBID-MS) at ultrasoft conditions (Fig. 1C). As shown previously detergents and phospholipids are still bound to the ionized complexes under these conditions and the mass of the protein portion is $40-50 \mathrm{kDa}$ lower [8], corresponding to the lower mass onset of the peak at about $808 \mathrm{kDa}$ (blue ticks, Fig. 1C).

Consistent with a reduction in molecular mass the specific NADH:hexammineruthenium (HAR) oxidoreductase activity of the subcomplex I $\delta$ was $90 \pm 4 \mu \mathrm{mol} \cdot \mathrm{min}^{-1} \cdot \mathrm{mg}^{-1}$ as compared to $65 \pm 2$ $\mu \mathrm{mol} \cdot \mathrm{min}^{-1} \cdot \mathrm{mg}^{-1}$ for purified holo-complex I. The non-physiological assay is indicative for a functional N-Module, as it solely depends on the presence of an intact NADH-oxidation site and FMN [41]. In contrast, inhibitor-sensitive NADH:ubiquinone oxidoreductase activity using ubiquinone analoga such as decylubiquinone (DBQ) or ubiquinone-1 $\left(\mathrm{Q}_{1}\right)$ was completely abolished in the subcomplex and could not be recovered by addition of lipids (see Supplementary Table I). This suggested that either the Q-module itself or the access path to its Q-binding pocket was affected. EPR spectroscopy indicated that a largely intact peripheral arm was present in the subcomplex (Fig. 2). The spectral signatures of iron-sulfur clusters N1, N3 and N4 appeared essentially unchanged. However, iron-sulfur cluster N2 showed a slight distortion in peak shape of the $g_{z}$ signal and a shift of the $g_{x y}$ signal suggesting a somewhat modified environment for this redox center as observed previously for bovine subcomplex I $\lambda[19]$.

Next we asked which of the central subunits had been retained in the subcomplex. Determining the subunit composition of a membrane integral multiprotein complex is a difficult task and we therefore applied three complementary techniques, dSDS-PAGE, LILBID-MS, and ESI-MS/MS of individual subunits and, if necessary, in addition immunological and molecular genetics approaches. Please note that we use bovine nomenclature for central subunits throughout (Table I). Fig. 3 shows silver-stained dSDS-PAGE and Fig. 4 LILBID-subunit spectra of complex I and subcomplex I $\delta$. LILBID mass spectrometry at high laser intensity dissociates non-covalently bound subunits of enzyme complexes and generates a complete mass fingerprint in a single experiment [8]. The analysis of these data and the results from ESI-MS/MS analysis of subcomplex I $\delta$ monomer and oligomers excised from BNgels are summarized in supplementary Table II. By all three techniques, the seven central subunits of the peripheral arm and subunits ND4 and ND5 of the membrane were detected in the subcomplex, while the hydrophobic subunits ND1, ND3 where clearly absent (Table I). The presence of the 49-kDa and PSST subunit was further confirmed by ELISA (see Supplementary Fig. 1). In intact complex I, subunits ND6 and ND4L could only be detected reliably by LILBID-MS. However, for ND6 $\left(\mathrm{M}_{\mathrm{r}}=\right.$ $20.76 \mathrm{kDa})$ this required shifting the almost identical mass of accessory subunit NUJM $\left(\mathrm{M}_{\mathrm{r}}=20.83\right.$ $\mathrm{kDa}$ ) by a strep-tag. This separated the two peaks in the spectrum of the tagged complex I and allowed unambiguous identification of both subunits and indicated that ND6 was not missing in the subcomplex (Fig. 4, insert). In contrast, the LILBID-spectra showed that ND4L had been removed. The spot for subunit ND2 partially overlapped with that of subunit ND4 in dSDS-PAGE, but it appeared that this subunit was at least markedly reduced in the subcomplex (Fig. 3). This was confirmed by LILBID-MS showing that subunit ND2 had been removed partially by the LDAO treatment (Fig. 4, peak 9).

With subunits ND1, ND3 and ND4L a significant portion of the proximal part of the $\mathrm{P}_{\mathrm{P}}$-module was completely missing in the subcomplex (Table I). As subunit ND1 is the central subunit that according to the recently published X-ray structural analysis of bacterial complex I [10] forms the major connection between the membrane arm and the peripheral arm, we concluded that in mitochondrial complex I another connection between the two parts must exist. Electron microscopic analysis of subcomplex I $\delta$ showed particles of the expected size with a three-domain substructure. Unfortunately, the very high flexibility of the particles limited the 3D structural analysis of the complex to very low resolutions of $\sim 60 \AA$ only (results not shown), precluding a more detailed interpretation.

\section{Subcomplex I $\delta$ lacks seven accessory subunits}

To determine which of the accessory subunits were missing in the subcomplex we again applied dSDS-PAGE, LILBID-mass spectrometry, and ESI-MS/MS (Figs. 3 and 4; see Supplementary Table 
II). For most proteins the results obtained with the three methods complemented each other. E.g. subunits with similar masses that resulted in overlapping peaks in LILBID-MS, were well enough separated in dSDS-PAGE. Only in the case of subunit NUJM obtaining an unambiguous result required using the complex I variant where this subunit was modified with a strep-tag. As discussed above subunit NUJM has a molecular mass almost identical to that of the central subunit ND6 resulting in overlapping peaks in LILBID-MS. In dSDS-PAGE the spot for subunit NUJM overlapped with that of subunit PSST making it again impossible to decide whether it was present in subcomplex I $\delta$. The spot for strep-tagged subunit NUJM was shifted away from that of subunit PSST but now partly overlapped with the spot corresponding to subunit NUPM (Fig. 3, insert 1). However, as subunit NUPM was detached by the LDAO treatment, as clearly evident from LILBID-MS, the remaining spot at this position could be assigned unambiguously to strep-tagged NUJM subunit in subcomplex I $\delta$ (Fig. 3, insert 2, and Western Blot data not shown). Peptides indicating the presence of subunit NUXM were found in the oligomeric form of subcomplex I $\delta$ by ESI-MS/MS. Employing ESI-MS/MS we confirmed the presence of the recently discovered subunit NUUM in the holo-enzyme and in subcomplex $I \delta[42]$. However, we derived a different splice pattern for this subunit with a longer Nterminal sequence (see Supplementary Table III).

To further confirm the presence or absence of individual subunits, ELISA experiments using specific monoclonal antibodies against the NUPM, NIAM and NESM subunits were performed. In contrast to the anti-NUPM antibody all other applied antibodies detected the respective subunit in the subcomplex (see Supplementary Table II and Supplementary Fig. 1). Overall, the evidence generated by dSDSPAGE, LILBID-, BN-PAGE/ESI-mass spectrometry and ELISA on the subunit composition of complete complex I and subcomplex I $\delta$ indicated that the seven accessory subunits NUPM, NUXM, NB6M, NIPM, NIMM, NI9M and ST1 were detached by the LDAO treatment of holo-complex I (Table I).

\section{Subunit ND2 is present in a subpopulation of subcomplex I $\delta$}

The total mass of the subunits not found in subcomplex $\mathrm{I} \delta$ was $177.4 \mathrm{kDa}$. This corresponds to a residual mass of the subcomplex of $786.3 \mathrm{kDa}$. As we have indications for the presence of at least one small yet uncharacterized subunit (Nübel et al., in preparation) this fits well to the total mass of $\sim 808$ $\mathrm{kDa}$ determined experimentally by LILBID-MS (blue ticks, Fig. 1C). However, this includes $53.3 \mathrm{kDa}$ of subunit ND2 that was found to be present only in a fraction of the subcomplex I $\delta$ sample. Closer inspection revealed that indeed a second peak series corresponding to a mass of $\sim 755 \mathrm{kDa}$ was present in the LILBID-MS spectra obtained in ultrasoft mode (red ticks, Fig. 1C). The difference of the experimentally determined masses is consistent with a subcomplex that in addition to the other ten subunits had lost subunit ND2. However, the peak intensities for the $808 \mathrm{kDa}$ species suggested a much higher fraction of complexes containing this subunit, than was expected based on LILBID-MS single subunit spectra and dSDS-PAGE (Figs. 3 and 4). A straightforward explanation for this discrepancy is that the subcomplexes lacking subunit ND2 were much less stable under the conditions of the LILBID experiment and for the most part were disintegrated even in the ultrasoft LILBID-mode making the $808 \mathrm{kDa}$ subcomplex the dominant species in the spectra. The observed series of peaks indicative of a mass of $413 \mathrm{kDa}$ can be consistently explained by decomposition of laser irradiation sensitive subcomplex If into the peripheral arm (green ticks, $\mathrm{Q} / \mathrm{N}$ module) and smaller fragments that are not resolved in the spectrum. We conclude that subunit ND2 was present only in a smaller fraction of the sample that however was more resistant towards laser irradiation and thus appeared as the major spectral component.

The arrangement of the accessory subunits can be deduced by combining biochemical structural and sequence data.

Subcomplex I $\delta$ lacked those central subunits of the membrane arm that according to the X-ray data $[10 ; 11]$ form the major interface with the peripheral arm. Yet it was stable enough to be prepared 
biochemically suggesting that most likely accessory subunits are involved in connecting the two major parts of mitochondrial complex I. We therefore compiled the available information on the 28 accessory subunits of $Y$. lipolytica complex I to assign them to the different domains (Table I) and find out how they may stabilize the core of central subunits of the P-module and how the P-module could be interconnected with the peripheral arm.

From their presence in the hydrophilic subcomplex I $\lambda$ [18-20;22] seven hydrophilic accessory subunits could be assigned to the Q- and N-module of the peripheral arm (Table I).

The sulfurtransferase subunit ST1 is not predicted to be imported into mitochondria (Table I) and therefore most likely resides in the intermembrane space. Because it is only loosely attached to complex I and has not been found in other species [43] we did not consider it here any further.

Of the remaining 20 accessory subunits 12 contain at least one predicted transmembrane segment identifying them as integral membrane proteins of the P-module [6] (Table I). We then reasoned that a high probability for mitochondrial import and the presence of a mitochondrial targeting sequence should indicate that a subunit resides on the matrix side of the membrane arm. This was clearly the case for the acyl carrier protein like subunits ACPM1 and ACPM2. For subunit NI2M the MITOPROT algorithm predicts a targeting sequence and a $100 \%$ import probability. However, we could unambigously identify the N-terminal peptide of the precursor protein just lacking the initial methionine by ESI-MS/MS (Supplementary Table III). We concluded that NI2M was also imported into the mitochondrial matrix, but that for some unknown reason the targeting sequence was not cleaved off. For the remaining five accessory subunits the MITOPROT algorithm predicted a very low import probability score and no mitochondrial targeting sequence. This suggested that they are probably located on the side of the membrane arm facing the intermembrane space. Proteins imported to the intermembrane space are characterized by internal targeting signals and specific patterns of cysteine residues [44]. Indeed, three of the subunits, NUPM, NIPM, and NB8M, exhibited canonical twin $\mathrm{Cx}_{9} \mathrm{C}$ motifs (see Supplementary Fig. 2). This indicated import to the intermembrane space via the Mia40 pathway [45]. In subunit NUPM the twin $\mathrm{Cx}_{9} \mathrm{C}$ motif is repeated. Also subunit NIDM may be imported via this pathway, although it contains only one pair of cysteine residues with a spacing of ten residues. NB4M contains no cysteine motif and it remained unclear how it reaches the intermembrane space side of complex I.

Next we assigned the 20 accessory subunits of the membrane arm to the different subdomains defined by X-ray structural analysis [11]. With the exception of subunits NEBM, NUXM, NUNM, and NUUM all subunits have orthologs in bovine complex I. Thus information from biochemically prepared subcomplexes I $\alpha$, I $\beta$ and I $\lambda$ of the bovine enzyme [18-20;22] subcomplexes of $Y$. lipolytica [22] (Dröse et al, submitted and Nübel et al, in preparation) and the proteomic analysis of subcomplex I $\delta$ reported here was included in this analysis.

Bovine subcomplex $\mathrm{I} \beta$ corresponds to the $\mathrm{P}_{\mathrm{D}}$-module of the membrane arm comprising central subunits ND4 and ND5 [18;21]. As it contains homologues of the single transmembrane domain (STMD) subunits [6] NIAM, NESM and NB2M and the hydrophilic accessory subunits NIDM, NI2M, and NB8M we can assign these subunits to the distal membrane arm module of $Y$. lipolytica complex I (Fig. 5). Bovine subunit B15, the homologue of $Y$. lipolytica NB5M, is detected in subcomplexes I $\beta$ and I $\alpha$, but it is, like STMD subunit NUNM absent in a $Y$. lipolytica subcomplex comprising the peripheral arm and the $\mathrm{P}_{\mathrm{P}}$-module (Nübel et al., in preparation). This suggested that these two subunits are located in the $\mathrm{P}_{\mathrm{D}^{-}}$-module, with subunit NB5M residing near its interface with the $\mathrm{P}_{\mathrm{P}}$-module. The homologue of the transmembrane subunit NUJM has been found in bovine subcomplex I $\lambda$ suggesting a direct connection with the peripheral arm. On the other hand, deletion of subunit NB8M in $Y$. lipolytica complex I was found to generate a subcomplex concomitantly lacking the $\mathrm{P}_{\mathrm{D}}$ module and subunit NUJM (Dröse et al, submitted). We thus assigned a position in the $\mathrm{P}_{\mathrm{D}}$ module at the interface to the peripheral arm and the $\mathrm{P}_{\mathrm{P}}$ module. Considering the absence of intermembrane space subunit NIPM in the two Y. lipolytica subcomplexes described above (Nübel et al, in preparation; Dröse et al, submitted) while the bovine homologue has been assigned to subcomplex I $\alpha$ [20] we suggest a position at the interface of the $\mathrm{P}_{\mathrm{P}}$ and $\mathrm{P}_{\mathrm{D}}$ module. In contrast to all other species analysed so far complex I from $Y$. lipolytica contains two subunits qualifying as mitochondrial acyl carrier proteins. ACPM1 is more similar to the bovine ortholog SDAP that was also detected in subcomplexes I $\beta$ and 
I $\alpha$. In the $Y$. lipolytica subcomplex lacking the $\mathrm{P}_{\mathrm{D}}$-module (Nübel et al., in preparation), ACPM1 is retained and ACPM2 is missing. We thus assigned ACPM1 to the $\mathrm{P}_{\mathrm{P}}$-module and ACPM2 to the $\mathrm{P}_{\mathrm{D}^{-}}$ module and propose that they reside at the interface between of the two domains. Taken together, we concluded that the $\mathrm{P}_{\mathrm{D}}$-module consists of 14 proteins, the two central subunits ND4 and ND5, seven accessory subunits with single or multiple transmembrane helices, and two hydrophilic accessory subunits at the matrix side and three at the intermembrane space side of complex I (Fig. 5).

Including subunit ACPM1, this leaves five central and eight accessory subunits for the $\mathrm{P}_{\mathrm{P}}$-module. The results obtained here by proteomic analysis of subcomplex $I \delta$ provided further information on the arrangement of these subunits within the $\mathrm{P}_{\mathrm{P}}$-module. The absence of the four membrane integral subunits NUXM, NB6M, NIMM, NI9M and the cysteine-containing subunit NUPM from subcomplex I $\delta$ identified these proteins, together with the central subunits ND1, ND3 and ND4L, as constituents of the proximal part of the $\mathrm{P}_{\mathrm{P}}$-module located directly under the peripheral arm (Fig. 5). The predicted position of subunit NUPM at the intermembrane space side of complex I could be confirmed by electron microscopy. The monoclonal antibody $31 \mathrm{~A} 8$ of the IgG subtype had been shown to bind with high affinity to complex I from Y. lipolytica in native and denaturing ELISA variants [46]. However, it failed to recognize any complex I polypeptide in a standard denaturing dSDS-PAGE/Western blot analysis. In contrast, under milder BN-dSDS-PAGE (3D-PAGE) conditions the integrity of the presumably discontinuous epitope was sufficiently conserved to allow binding of the antibody to the NUPM subunit (Fig. 3, insert 3). The identity of the corresponding spot in dSDS-PAGE was confirmed by ESI-MS/MS (data not shown). When we analyzed the co-complex of complex I and antibody $31 \mathrm{~A} 8$ by electron microscopic single particle analysis (Fig. 6), the mass corresponding to the antibody was clearly connected to the proximal end of the membrane arm on the intermembrane space side. This confirmed the predicted position of subunit NUPM. Together with the central subunits ND6 and ND2, the remaining two accessory subunits, the membrane integral subunit NEBM and the subunit NB4M on the intermembrane space side, could be assigned to the part of the $\mathrm{P}_{\mathrm{P}}$-module oriented towards the $\mathrm{P}_{\mathrm{D}}$-module (Fig. 5).

\section{DISCUSSION}

Despite recent progress in X-ray crystallographic analysis of mitochondrial complex I [11] much of the architecture of this membrane integral multiprotein complex of $>40$ different subunits remains unknown. Notably, the arrangement of the numerous accessory subunits, and how it may relate to the function and stability of complex I remained obscure. Here we report the preparation and proteomic analysis of subcomplex I $\delta$ of complex I providing clues on how the peripheral and the membrane arm are connected.

The most remarkable feature of subcomplex I $\delta$ was that it lacked subunits ND1 and ND3. From X-ray structural analysis of bacterial complex I [10] and cross linking experiments [47] it seemed that these two central subunits are the only proteins of the membrane arm making direct connection to the peripheral arm via the 49-kDa and PSST subunits where the extended ubiquinone binding pocket has been located by site-directed mutagenesis [48]. The observation that electron transfer to ubiquinone was completely abolished and that the EPR signature of the electron donor for ubiquinone, iron-sulfur cluster N2 was slightly altered in subcomplex I $\delta$ fits to inhibitor labelling studies suggesting that ND1 might be important for guiding ubiquinone to the active site [49;50]. The fact that subcomplex I $\delta$ still contained the complete Q- and N-module and much of the P-module (Fig. 5B) indicated that additional connections between the two arms of mitochondrial complex I must exist.

To obtain further insight into the arrangement of the accessory subunits we performed a sequence based analysis to predict the import pathways and the sub-compartment localization of each subunit (Table I). Remarkably, three complex I subunits, NUPM, NIPM and NB8M contain $\mathrm{CX}_{9} \mathrm{C}$ motifs that are a hallmark of proteins imported to the intermembrane space by the Mia40 pathway [45;51] (see Supplementary Fig. 2). The sulfhydryl oxidase Erv1 and the redox-activated import acceptor Mia40 form a disulfide relay system which is located in the intermembrane space of mitochondria from fungi, plants and animals. Y. lipolytica protein YALI0E21373g (Genolevures data bank) is similar to 
Saccharomyces cerevisiae Mia40 (Uniprot accession number P36046) and protein YALI0D25894g (Genolevures data bank) is similar to S. cerevisiae Erv1 Flavin-linked sulfhydryl oxidase (P27882) indicating the presence of Erv1 and Mia40 as part of a disulfide relay system in Y. lipolytica that promotes retention of imported proteins in the intermembrane space. We consider it remarkable that coordination of complex I assembly not only involves two genomes, as complex I subunits are encoded by nuclear and mitochondrial DNA, but also multiple import pathways that direct nuclear encoded subunits to either the matrix or intermembrane space side.

Based on the predictions derived from this sequence analysis and the results obtained for subcomplex I $\delta$ together with earlier results obtained with other subcomplexes of complex I from bovine heart and $Y$. lipolytica, we developed a model for the arrangement of the central and accessory subunit of the membrane arm or P-module of complex I (Fig. 5). We compared our model with the electron density map of complex I from Y. lipolytica (Fig. 7) [11]. In line with our model electron density on the matrix side of the membrane arm is rather limited and concentrated in the distal membrane arm protrusion (DMP) that had been already identified by electron microsocopy [52]. The DMP may in part correspond to subunit NI2M and the N-terminal hydrophilic domains of STMD subunits NIAM and NESM [6]. In contrast, a prominent layer of electron density at the intermembrane space side harbours a number of elongated density features probably corresponding to $\alpha$-helices oriented in parallel to the membrane plane (Fig. 7). This fits well to our prediction that subunits NUPM, NB4M, NIPM, NB8M, and NIDM are arranged along this side of complex I. We propose that this ensemble of accessory subunits functions as a platform stabilizing the membrane arm that is connected via the peripherally arranged transmembrane segments of the eleven membrane integral accessory subunits to the matrix side of complex I. Thereby, these accessory subunits seem to form a scaffold around the central subunits of the $\mathrm{P}$-module. Our observation that the $\mathrm{P}_{\mathrm{D}}$-module stayed connected to the peripheral arm in subcomplex I $\delta$ even in the absence of subunit ND2, suggested that subunit NUJM may be of particular importance to keep the peripheral arm connected to the residual membrane arm. Remarkably, this scaffold of accessory subunits seemed to be partially retained even under conditions that detached several central subunits from the membrane arm. It is tempting to speculate that the pronounced sensitivity of the central subunits in the proximal membrane arm domain towards disintegration reflects a functionally relevant flexibility inherent to a conformational coupling mechanism.

\section{Author contribution}

H.A. designed and performed research, produced complex I and subcomplex samples, performed activity assays, BN-PAGE, dSDS-PAGE, ELISA and alignments, analyzed data, wrote the paper; K.Z. performed EPR spectroscopy; Z.W. performed western blot from 3D-PAGE; E.N. identified subunit NEBM; H.H. and M.S. performed ESI-MS/MS, analyzed data; S.K. provided NUJM-streptag; B.B. and L.S. performed LILBID-MS, analyzed data; M.R. performed EM single particle analysis; U.B. and V.Z. analyzed data, wrote the paper.

\section{Acknowledgements}

We thank Ilka Wittig and Hermann Schägger for fruitful discussions and Andrea Duchene, Karin Siegmund, Maximilian Mattil and Gudrun Beyer for excellent technical assistance. We thank Stefan Kerscher for analysis of the splice pattern of subunit NUUM.

\section{Funding}

This work was supported by the Deutsche Forschungsgemeinschaft, grant ZI 552/3-1 to V.Z., the Cluster of Excellence Frankfurt "Macromolecular Complexes" at the Goethe University Frankfurt (EXC115) and NIH grant 2RO1 GM068650 to M.R.. 


\section{References}

1 Yagi,T. and Matsuno-Yagi,A. (2003) The proton-translocating NADH-quinone oxidoreductase in the respiratory chain: the secret unlocked. Biochemistry, 42, 2266-2274.

2 Zickermann,V., Kerscher,S., Zwicker,K., Tocilescu,M.A., Radermacher,M. and Brandt,U. (2009) Architecture of complex I and its implications for electron transfer and proton pumping. Biochimica et Biophysica Acta-Bioenergetics, 1787, 574-583.

3 Brandt,U. (2006) Energy converting NADH : Quinone oxidoreductase (Complex I). Annu.Rev.Biochem., 75, 69-92.

4 Lin,M.T. and Beal,M.F. (2006) Mitochondrial dysfunction and oxidative stress in neurodegenerative diseases. Nature, 443, 787-795.

5 McKenzie,M. and Ryan,M.T. (2010) Assembly factors of human mitochondrial complex I and their defects in disease. IUBMB Life, 62, 497-502.

6 Zickermann,V., Angerer,H., Ding,M., Nübel,E. and Brandt,U. (2010) Small single transmembrane domain (STMD) proteins organize the hydrophobic subunits of large membrane protein complexes. FEBS Lett., 584, 2516-2525.

7 Hirst,J., Carroll,J., Fearnley,I.M., Shannon,R.J. and Walker,J.E. (2003) The nuclear encoded subunits of complex I from bovine heart mitochondria. Biochim.Biophys.Acta, 1604, 135-150.

8 Morgner,N., Zickermann,V., Kerscher,S., Wittig,I., Abdrakhmanova,A., Barth,H.D., Brutschy,B. and Brandt,U. (2008) Subunit mass fingerprinting of mitochondrial complex I. Biochimica et Biophysica Acta-Bioenergetics, 1777, 1384-1391.

9 Clason,T., Ruiz,T., Schagger,H., Peng,G., Zickermann,V., Brandt,U., Michel,H. and Radermacher,M. (2010) The structure of eukaryotic and prokaryotic complex I. J.Struct.Biol., 169, 81-88.

10 Efremov,R.G., Baradaran,R. and Sazanov,L.A. (2010) The architecture of respiratory complex I. Nature, 465, 441-445.

11 Hunte,C., Zickermann,V. and Brandt,U. (2010) Functional modules and structural basis of conformational coupling in mitochondrial complex I. Science, 329, 448-451.

12 Sazanov,L.A. and Hinchliffe,P. (2006) Structure of the hydrophilic domain of respiratory complex I from Thermus thermophilus. Science, 311, 1430-1436.

13 Zickermann,V., Bostina,M., Hunte,C., Ruiz,T., Radermacher,M. and Brandt,U. (2003) Functional implications from an unexpected position of the $49 \mathrm{kDa}$ subunit of complex I. J.Biol.Chem., 278, 29072-29078.

14 Clason,T., Zickermann,V., Ruiz,T., Brandt,U. and Radermacher,M. (2007) Direct localization of the 51 and $24 \mathrm{kDa}$ subunits of mitochondrial complex I by three-dimensional difference imaging. J.Struct.Biol., 159, 433-442.

15 Leif,H., Sled,V.D., Ohnishi,T., Weiss,H. and Friedrich,T. (1995) Isolation and characterization of the proton-translocating NADH:ubiquinone oxidoreductase from Escherichia coli. Eur.J.Biochem., 230, 538-548. 
16 Yip,C.Y., Harbour,M.E., Jayawardena,K., Fearnley,I.M. and Sazanov,L.A. (2010) Evolution of respiratory complex I: 'supernumerary' subunits are present in the $\{$ alpha\}-proteobacterial enzyme. J Biol Chem., 286, 5023-33

17 Peng,G., Fritzsch,G., Zickermann,V., Schägger,H., Mentele,R., Lottspeich,F., Bostina,M., Radermacher,M., Huber,R., Stetter,K.O. and Michel,H. (2003) Isolation, characterization and electron microscopic single particle analysis of the NADH:ubiquinone oxidoreductase (complex I) from the hyperthermophilic eubacterium Aquifex aeolicus. Biochemistry, 42, 3032-3039.

18 Finel,M., Skehel,J.M., Albracht,S.P.J., Fearnley,I.M. and Walker,J.E. (1992) Resolution of NADH:ubiquinone oxidoreductase from bovine heart mitochondria into two subcomplexes, one of which contains the redox centers of the enzyme. Biochemistry, 31, 11425-11434.

19 Finel,M., Majander,A.S., Tyynelä,J., de Jong,A.M.P., Albracht,S.P.J. and Wikström,M.K.F. (1994) Isolation and characterisation of subcomplexes of the mitochondrial NADH:ubiquinone oxidoreductase (complex I). Eur.J.Biochem., 226, 237-242.

20 Carroll,J., Fearnley,I.M., Shannon,R.J., Hirst,J. and Walker,J.E. (2003) Analysis of the subunit composition of complex I from bovine heart mitochondria. Mol.Cell.Proteomics, 2, 117-126.

21 Sazanov,L.A., Peak-Chew,S.Y., Fearnley,I.M. and Walker,J.E. (2000) Resolution of the membrane domain of bovine complex I into subcomplexes: implications for the structural organization of the enzyme. Biochemistry, 39, 7229-7235.

22 Abdrakhmanova,A., Zickermann,V., Bostina,M., Radermacher,M., Schägger,H., Kerscher,S. and Brandt,U. (2004) Subunit composition of mitochondrial complex I from the yeast Yarrowia lipolytica. Biochim.Biophys.Acta, 1658, 148-156.

23 Kashani-Poor,N., Kerscher,S., Zickermann, V and Brandt,U. (2001) Efficient large scale purification of his-tagged proton translocating NADH : ubiquinone oxidoreductase (complex I) from the strictly aerobic yeast Yarrowia lipolytica. Biochim.Biophys.Acta, 1504, 363-370.

24 Grgic,L., Zwicker,K., Kashani-Poor,N., Kerscher,S. and Brandt,U. (2004) Functional significance of conserved histidines and arginines in the $49 \mathrm{kDa}$ subunit of mitochondrial complex I. J.Biol.Chem., 279, 21193-21199.

25 Tocilescu,M.A., Fendel,U., Zwicker,K., Kerscher,S. and Brandt,U. (2007) Exploring the ubiquinone binding cavity of respiratory complex I. J.Biol.Chem., 282, 29514-29520.

26 Wittig,I., Velours,J., Stuart,R. and Schagger,H. (2008) Characterization of domain interfaces in monomeric and dimeric ATP synthase. Molecular \& Cellular Proteomics, 7, 995-1004.

27 Nübel,E., Wittig,I., Kerscher,S., Brandt,U. and Schägger,H. (2009) Two-dimensional native electrophoretic analysis of respiratory supercomplexes from Yarrowia lipolytica, Proteomics, 9 , 2408-18

28 Rais,I., Karas,M. and Schägger,H. (2004) Two-dimensional electrophoresis for the isolation of integral membrane proteins and mass spectrometric identification. Proteomics, 4, 2567-2571.

29 Morgner,N., Barth,H.D. and Brutschy,B. (2006) A new way to detect noncovalently bonded complexes of biomolecules from liquid micro-droplets by laser mass spectrometry. Austral.J.Chem., 59, 109-114.

30 Collins,M.O., Yu,J.S. and Choudhary,J.S. (2008) Analysis protein complexes by 1D-SDS-PAGE and tandem mass spectrometry. nature protocols. doi: 10.1038/nprot.2008.123 
31 Schafer,A., Zick,M., Kief,J., Steger,M., Heide,H., Duvezin-Caubet,S., Neupert,W. and Reichert,A.S. (2010) Intramembrane proteolysis of Mgm1 by the mitochondrial rhomboid protease is highly promiscuous regarding the sequence of the cleaved hydrophobic segment. J Mol Biol, 401, 182-193.

32 Stoops,J.K., Kolodziej,S.J., Schroeter,J.P., Bretaudiere,J.P. and Wakil,S.J. (1992) Structurefunction relationships of the fatty acid synthase: negative stain, cryo -electron microscopy, and image analysis studies of the end views of the structure. Proc.Natl.Acad.Sci.USA, 89, 6585-6589.

33 Ruiz,T. and Radermacher,M. (2006) Three-dimensional analysis of single particles by electron microscopy: sample preparation and data acquisition. Methods Mol Biol, 319, 403-425.

34 Radermacher,M. (1997) Radon transform techniques for alignment and 3D reconstruction from random projections. Scanning Microscopy, 11, 171-177.

35 van Heel,M. and Frank,J. (1981) Use of multivariate statistics in analysing the images of biological macromolecules. Ultramicroscopy, 6, 187-194.

36 Diday, E. (1971) La methode de nuees dynamiques. Rev.Stat.App1. (19), 19-33.

37 Claros,M.G. and Vincens,P. (1996) Computational method to predict mitochondrially imported proteins and their targeting sequences. Eur.J.Biochem., 241, 779-786.

38 Larkin,M.A., Blackshields,G., Brown,N.P., Chenna,R., McGettigan,P.A., McWilliam,H., Valentin,F., Wallace,I.M., Wilm,A., Lopez,R., Thompson,J.D., Gibson,T.J. and Higgins,D.G. (2007) Clustal W and Clustal X version 2.0. Bioinformatics, 23, 2947-2948.

39 Krogh,A., Larsson,B., von Heijne,G. and Sonnhammer,E.L. (2001) Predicting transmembrane protein topology with a hidden Markov model: application to complete genomes. J.Mol.Biol., 305, $567-580$.

40 Tusnady,G.E. and Simon,I. (2001) The HMMTOP transmembrane topology prediction server. Bioinformatics, 17, 849-850.

41 Gavrikova,E.V., Grivennikova,V.G., Sled,V.D., Ohnishi,T. and Vinogradov,A.D. (1995) Kinetics of the mitochondrial three-subunit NADH dehydrogenase interaction with hexammineruthenium(III). Biochim.Biophys.Acta, 1230, 23-30.

42 Bridges,H.R., Fearnley,I.M. and Hirst,J. (2010) The subunit composition of mitochondrial NADH:ubiquinone oxidoreductase (complex I) from Pichia pastoris. Molecular \& Cellular Proteomics, 9, 2318-2326.

43 Abdrakhmanova,A, Dobrynin,K., Zwicker,K., Kerscher,S. and Brandt,U. (2005) Functional sulfurtransferase is associated with mitochondrial complex I from Yarrowia lipolytica, but is not required for assembly of its iron-sulfur clusters. FEBS Lett., 579, 6781-6785.

44 Herrmann,J.M. and Koehl,R. (2007) Catch me if you can! Oxidative protein trapping in the intermembrane space of mitochondria. Journal of Cell Biology, 176, 559-563.

45 Cavallaro,G. (2010) Genome-wide analysis of eukaryotic twin CX(9)C proteins. Mol Biosyst.. 6, 2459-70

46 Zickermann,V., Wumaier,Z., Wrzesniewska,B., Hunte,C. and Schagger,H. (2010) Native immunoblotting of blue native gels to identify conformation-specific antibodies. Proteomics, $\mathbf{1 0}$, 159-163. 
47 Kao,M.C., Matsuno-Yagi,A. and Yagi,T. (2004) Subunit proximity in the $\mathrm{H}^{+}$-translocating NADHquinone oxidoreductase probed by zero-length cross-linking. Biochemistry, 43, 3750-3755.

48 Tocilescu,M.A., Zickermann,V., Zwicker,K. and Brandt,U. (2010) Quinone binding and reduction by respiratory complex I. Biochim Biophys Acta., 1797, 1883-90

49 Schuler,F. and Casida,J.E. (2001) Functional coupling of PSST and ND1 subunits in NADH : ubiquinone oxidoreductase established by photoaffinity labeling. Biochim Biophys Acta, 1506, 7987.

50 Murai,M., Ishihara,A., Nishioka,T., Yagi,T. and Miyoshi,H. (2007) The ND1 subunit constructs the inhibitor binding domain in bovine heart mitochondrial complex I. Biochemistry, 46, 6409-6416.

51 Szklarczyk,R., Wanschers,B.F., Nabuurs,S.B., Nouws,J., Nijtmans,L.G. and Huynen,M.A. (2011) NDUFB7 and NDUFA8 are located at the intermembrane surface of complex I. FEBS Lett, 585, 737-743.

52 Radermacher,M., Ruiz,T., Clason,T., Benjamin,S., Brandt,U. and Zickermann,V. (2006) The threedimensional structure of complex I from Yarrowia lipolytica: A highly dynamic enzyme.

J.Struct.Biol., 154, 269-279.

53 Rosenthal,P.B. and Henderson,R. (2003) Optimal determination of particle orientation, absolute hand, and contrast loss in single-particle electron cryomicroscopy. J Mol Biol, 333, 721-745. 
B Biochemical Journal Immediate Publication. Published on 05 May 2011 as manuscript BJ20110359

\begin{tabular}{|c|c|c|c|c|c|c|c|c|c|c|}
\hline 15 & NUEM & $39-\mathrm{kDa}$ & 42,706 & 40,434 & 74 & no & yes[22] & NADPH & $\mathrm{Q} / \mathrm{N}$ & matrix \\
\hline 16 & ST 1 & & 34,621 & 34,490 & 27 & no & no & - Met $^{\mathrm{d}}$ & unknown & unknown \\
\hline 17 & NESM & ESSS & 28,436 & 23,438 & 87 & yes & yes[22] & STMD $^{e}$ & $\mathrm{P}_{\mathrm{D}}$ & membrane integral \\
\hline 18 & NUJM & B14.7 & 20,828 & 20,696 & 40 & no & & $\begin{array}{l}\text { - Met?, } 2 \mathrm{TMH}^{\mathrm{f}}, \\
\text { opt. + StreptagII }\end{array}$ & & ntegral \\
\hline 19 & NUZM & B14.5a & & 19,750 & 51 & yes & no [22] & & $\mathrm{Q} / \mathrm{N}$ & matrix \\
\hline 20 & NUPM & PGIV & 19,327 & 19,196 & 5 & no & no & Mia- $\mathrm{Cx}_{\mathrm{y}} \mathrm{C}^{\mathrm{g}}$, - Miet & $P_{P}$ & intermembrane side \\
\hline 21 & NUXM & & & 18,565 & 10 & no & & $\underset{\text { motif }^{2}}{2 \mathrm{TMH}, \mathrm{Zn}^{2+}}$ & $\mathrm{P}_{\mathrm{P}}$ & membrane integral \\
\hline 22 & N7BM & B17.2 & & 16,153 & 97 & no & & & $\mathrm{Q} / \mathrm{N}$ & matrix \\
\hline 23 & NUYM & AQDQ & 18,671 & 15,940 & 98 & yes & $\mathrm{s}[22]$ & & $\mathrm{Q} / \mathrm{N}$ & matrix \\
\hline 24 & NUFM & B13 & 16,635 & 15,573 & 91 & yes & yes [22] & & $\mathrm{Q} / \mathrm{N}$ & matrix \\
\hline 25 & NIAM & ASHI & 17,307 & 14,642 & 100 & yes & yes [22] & STMD & $\mathrm{P}_{\mathrm{D}}$ & membrane integral \\
\hline 26 & NB4M & B14 & 14,758 & 14,627 & & no & & - Met & $\mathrm{P}_{\mathrm{P}}$ & intermembrane side \\
\hline 27 & NB6M & B16.6 & 14,091 & 13,960 & 2 & no & no & - Met, STMD & $\mathrm{P}_{\mathrm{P}}$ & membrane integral \\
\hline 28 & NUNM & - & 15,795 & 13,301 & 94 & yes & & STMD & $\mathrm{P}_{\mathrm{D}}$ & membrane integral \\
\hline 29 & NUMM & $13-\mathrm{kDa}$ & 15,129 & $13,117^{b}$ & 97 & yes & & & $\mathrm{Q} / \mathrm{N}$ & matrix \\
\hline 30 & NI2M & B22 & 12,880 & 12,749 & 100 & yes & no & - Met & $\mathrm{P}_{\mathrm{D}}$ & matrix side \\
\hline 31 & NB8M & B18 & 11,199 & 11,068 & 1 & no & no & $\begin{array}{l}\text { Mia-Cx } x_{y} C,- \\
\text { Met[22] }\end{array}$ & $\mathrm{P}_{\mathrm{D}}$ & intermembrane side \\
\hline 32 & NIDM & PDSW & 11,021 & 10,890 & 1 & no & no & Mia-Cx $x_{y} C$, - Met & $\mathrm{P}_{\mathrm{D}}$ & intermembrane side \\
\hline
\end{tabular}


B Biochemical Journal Immediate Publication. Published on 05 May 2011 as manuscript BJ20110359

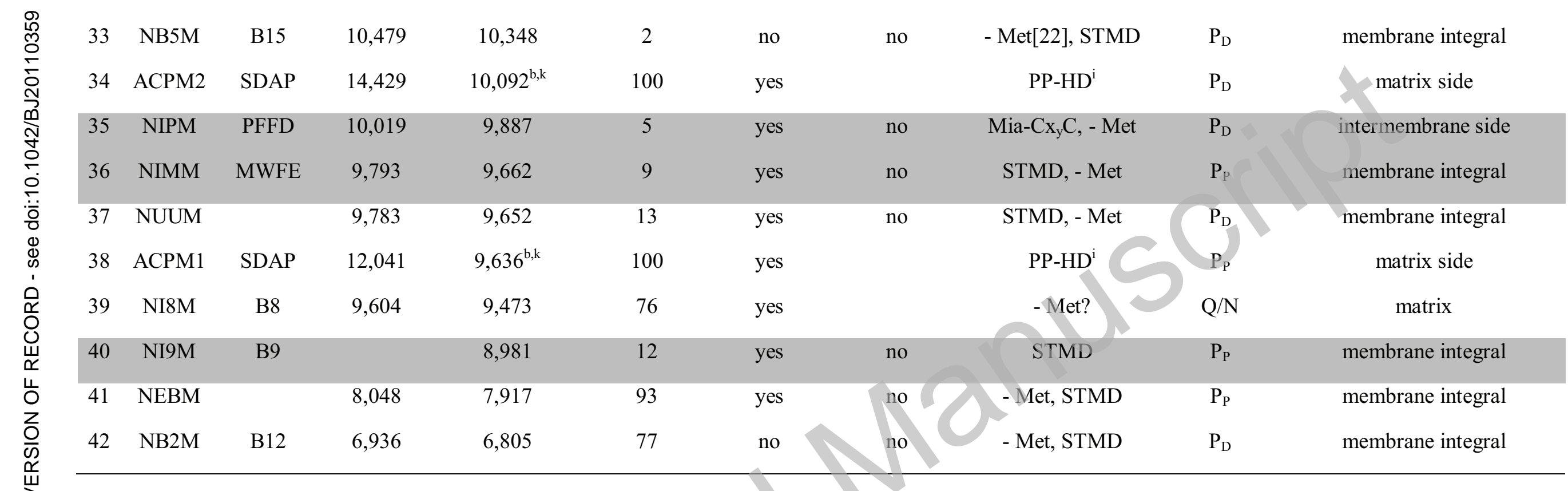

a Taken from [8] except for additional subunits NUUM, NEBM; and subunits ST1, NUMM, NI2M, NIPM and NI9M for which a corrected M $\mathrm{M}_{\mathrm{r}}$ based on new ESIMS/MS is given. Note that for calculation of the total mass of complexes comprising the intact $\mathrm{N}$ - and Q-module $2.918 \mathrm{kDa}$ have to be added for the mass of the eight iron-sulfur clusters and one FMN; for complexes containing subunit NUEM $0.745 \mathrm{kDa}$ have to be added to account for tightly bound NADPH [8].

${ }^{\mathbf{b}}$ Probability for import into mitochondria and presence of a targeting sequence was calculate using the MITOPROT algorithm [37].

${ }^{\mathbf{c}}$ The assignment is based on previous studies with biochemically prepared subcomplexes of bovine heart and Y. lipolytica complex I [22;52].

d -Met, N-terminal methionine is cleaved off; a question mark indicates that this processing has not been confirmed experimentally.

${ }^{\mathrm{e}} \mathrm{STMD}$, single transmembrane domain protein [6].

${ }^{\mathbf{f}} \mathrm{TMH}$, transmembrane helices as predicted by the TMHMM 2.0 algorithm (http://www.cbs.dtu.dk/services/TMHMM).

${ }^{\mathrm{g}}$ These proteins contain a cysteine-motif characteristic for proteins imported into the intermembrane space via the Mia-pathway [44].

${ }^{\mathbf{h}}$ NUXM contains a $\mathrm{Zn}^{2}$-binding motif (HSIAGLFIFPW) in the C-terminal loop region (PROSITE, Swiss Institute of Bioinformatics).

${ }^{\text {i }}$ PP-HD, Phosphopantetheine-hydroxy-tetradecanoate

${ }^{j}$ includes $\mathrm{M}_{\mathrm{r}}$ of His-tag

${ }^{k}$ includes of PP-HD $\left(\mathrm{M}_{\mathrm{r}}=565 \mathrm{Da}\right)$ 


\section{Figure legends}

\section{Figure 1 Exposure of native complex I to LDAO generates subcomplex I $\delta$}

(A) BN-PAGE of solubilized mitochondrial membranes from $Y$. lipolytica (left lane) and subcomplex I $\delta$ (right lane). The migration behavior of subcomplex $I \delta$ (I $\delta$ ) indicated a lower mass compared with native complex I (CI). Note that complex I subunit ST1 is detached under BN-PAGE conditions resulting in a total mass of $\sim 930 \mathrm{kDa}$ for the control; complex $\mathrm{V}$ monomer $(\mathrm{CV}, \sim 580 \mathrm{kDa}$ ), complex III dimer $\left(\mathrm{CIII}_{2}, \sim 420 \mathrm{kDa}\right)$. The bands in the high molecular weight range in the right lane were identified as oligomers of subcomplex $I \delta$ (n I $\delta$ ). (B) Subcomplex I $\delta$ (light grey) eluted as a symmetrical peak with slightly increased retention volume compared with native complex I (black) in size exclusion chromatography. (C) Non-destructive LILBID-MS analysis of subcomplex $I \delta$ at low laser intensity. A prominent peak series indicated $\mathrm{m} / \mathrm{z}$ values $(\mathrm{I} \delta+\mathrm{ND} 2$, blue) of $\sim 850 \mathrm{kDa}$ including detergent and lipids. As for native complex I the protein mass was found to be 40-50 kDa offset from the peak maximum and best approximated by evaluating $\mathrm{m} / \mathrm{z}$ values of the left peak edges the mass of subcomplex $\mathrm{I} \delta+\mathrm{ND} 2$ was determined to be $\sim 808 \mathrm{kDa}$ (blue ticks). A second peak series corresponding to $\sim 755 \mathrm{kDa}$ was assigned to subcomplex I $\delta$ without ND2 (I $\delta$, red ticks). A third peak series of $\sim 413 \mathrm{kDa}$ can be assigned to the peripheral arm $(\mathrm{Q} / \mathrm{N}$-module, green ticks) that is generated by disintegration of laser irradiation sensitive subcomplex $I \delta$ (red) (see text for details).

\section{Figure 2 EPR spectra of complex I and subcomplex $I \delta$}

Spectra were recorded using the following parameters: microwave frequency $9.47 \mathrm{GHz}$, modulation amplitude $0.64 \mathrm{mT}$, modulation frequency $100 \mathrm{kHz}$. Complex I or subcomplex $\mathrm{I} \delta(15 \mathrm{mg} / \mathrm{ml})$ were reduced with NADH $(2 \mathrm{mM})$ and the spectra were recorded at $12 \mathrm{~K}$. *Organic radical signal, probably representing an impurity.

\section{Figure 3 dSDS-PAGE of purified complex I (A) and subcomplex I $\delta$ (B)}

Subunits missing in subcomplex I $\delta$ are highlighted by a circle. In the subcomplex an extra spot was visible near the $49-\mathrm{kDa}$ subunit $(49-\mathrm{kDa} \Delta \mathrm{N})$. It was identified by ESI-MS/MS as a degradation product of the $49-\mathrm{kDa}$ subunit lacking a $3 \mathrm{kDa}$ fragment at the $\mathrm{N}$-terminus. Inserts 1 and 2 show the section of a dSDS-gel around subunit NUJM of complex I or subcomplex I $\delta$ prepared from a strain carrying a strep-tagged version of this subunit. Insert 3 shows a section of a Coomassie-stained BNdSDS-PAGE gel of complex I (left) and the corresponding Western blot (right). Subunit NUPM was recognized by monoclonal antibody (mab) 31 A8 (anti-NUPM). Note that the assignment for some of the accessory subunits was updated and is therefore different from [22].

\section{Figure 4 LILBID mass fingerprint spectra of complex I and subcomplex $\delta$}

The peaks are numbered according to Table I. Insert, detail from mass spectra of a complex I variant carrying a strep-tag on subunit NUJM (grey, dashed line) and the corresponding subcomplex I $\delta$ (black, dashed line). The mass shift permitted to identify subunits NUJM (18) and ND6 (13) in the subcomplex (asterisks). Taking into account new evidence on C-terminal peptides and prediction of targeting sequences (Table I) we reassigned the peaks for subunits NUMM and NI9M (compare [8]). The peak at $7.7 \mathrm{kD}$ a previously assigned to NI9M remains unassigned. To account for these changes and the newly discovered subunits NUUM and NEBM, the assignment of peaks 27 to 29 and peaks 37 to 42 was updated to match decreasing molecular masses of the corresponding subunits. The spectrum of subcomplex I $\delta$ exhibited a few additional peaks that correspond either to higher charged species of the $75-\mathrm{kDa}(1), 51-\mathrm{kDa}(2)$ and $49-\mathrm{kDa}(3)$ or to the truncated version of the $49-\mathrm{kDa}$ subunit $(3 \Delta \mathrm{N}$, see Figure 3).

Figure 5 Subunit arrangement in complex I (A) and subcomplex I $\delta$ (B) from Y. lipolytica Accessory subunits form an extended scaffold and connect the peripheral arm (Q/N-module, white) and the membrane arm $\left(\mathrm{P}_{\mathrm{P}}\right.$-module, light grey; $\mathrm{P}_{\mathrm{D}}$-module, dark grey). See text for details. The long helical transmission element [11] implicated in conformational energy transfer is shown in light grey. In the subcomplex subunit ND2 is shown transparent, because it was present in substoichiometric 
amounts. Subunit ST1 is not shown. The positions of subunits NB4M, NIDM and NI2M have not been confirmed experimentally.

Figure 6 Localization of the NUPM subunit on the intermembrane space side of the membrane arm

Two-dimensional average of single particle images of complex I decorated with monoclonal antibody 31A8 against the NUPM subunit. Alignment and classification of 749 images yielded six major classes. Class 3, shown here and containing 16\% (118) of the particles, most clearly shows the bound antibody. The resolution of the average is $30 \AA$ as measured by the Fourier Ring Correlation with a cutoff criterion of 0.3 [53]. Scale bar, $100 \AA$.

\section{Figure $7 \quad$ Membrane arm of mitochondrial complex I}

Section from electron density map (blue mesh) of $Y$. lipolytica complex $I$ and structural model for transmembrane segments (yellow) [11]. The contour line of a complex I monomer is tentatively indicated by a dashed blue line; the red line separates $\mathrm{P}_{\mathrm{P}}$ and $\mathrm{P}_{\mathrm{D}}$-module; DMP, distal membrane arm protrusion. A prominent continuous layer of extrinsic protein mass is attached to the intermembrane space side (IMS) of the membrane arm (compare Fig. 5). 
B Biochemical Journal Immediate Publication. Published on 05 May 2011 as manuscript BJ20110359

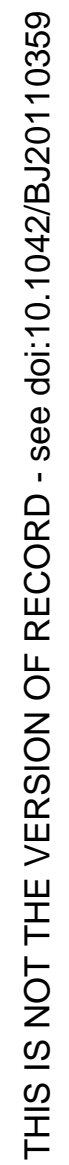
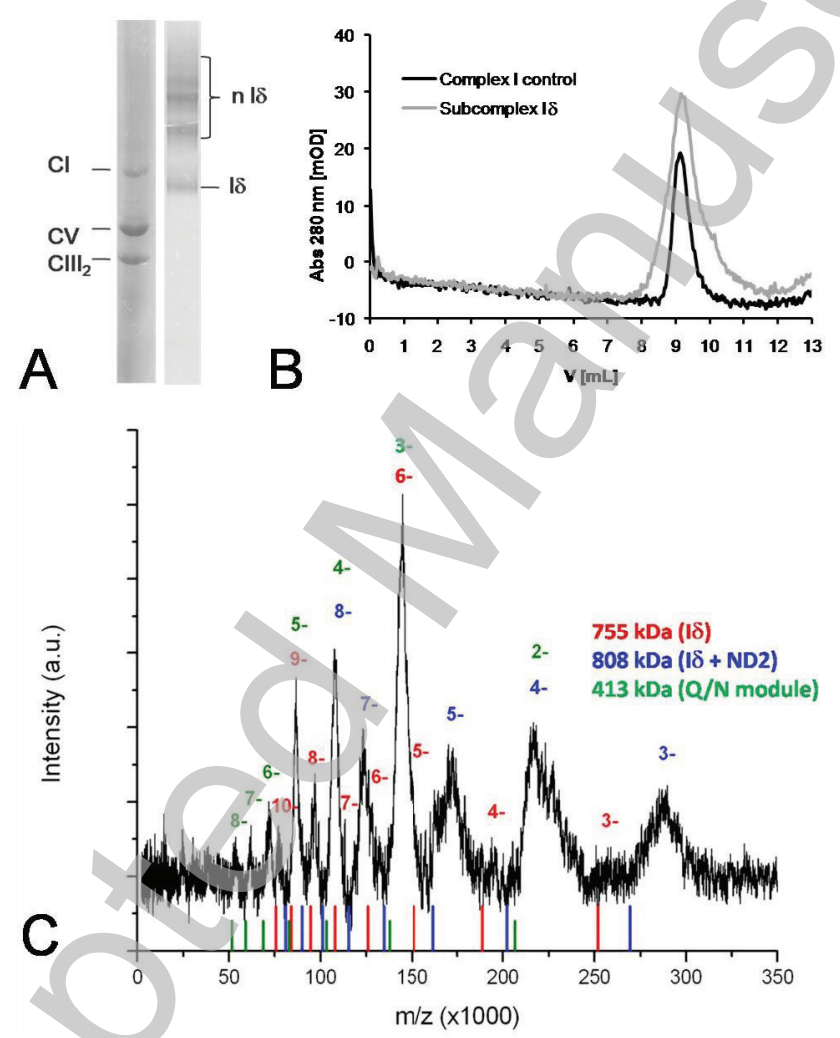

Licenced copy. Copying is not permitted, except with prior permission and as allowed by law. (c) 2011 The Authors Journal compilation (c) 2011 Portland Press Limited 


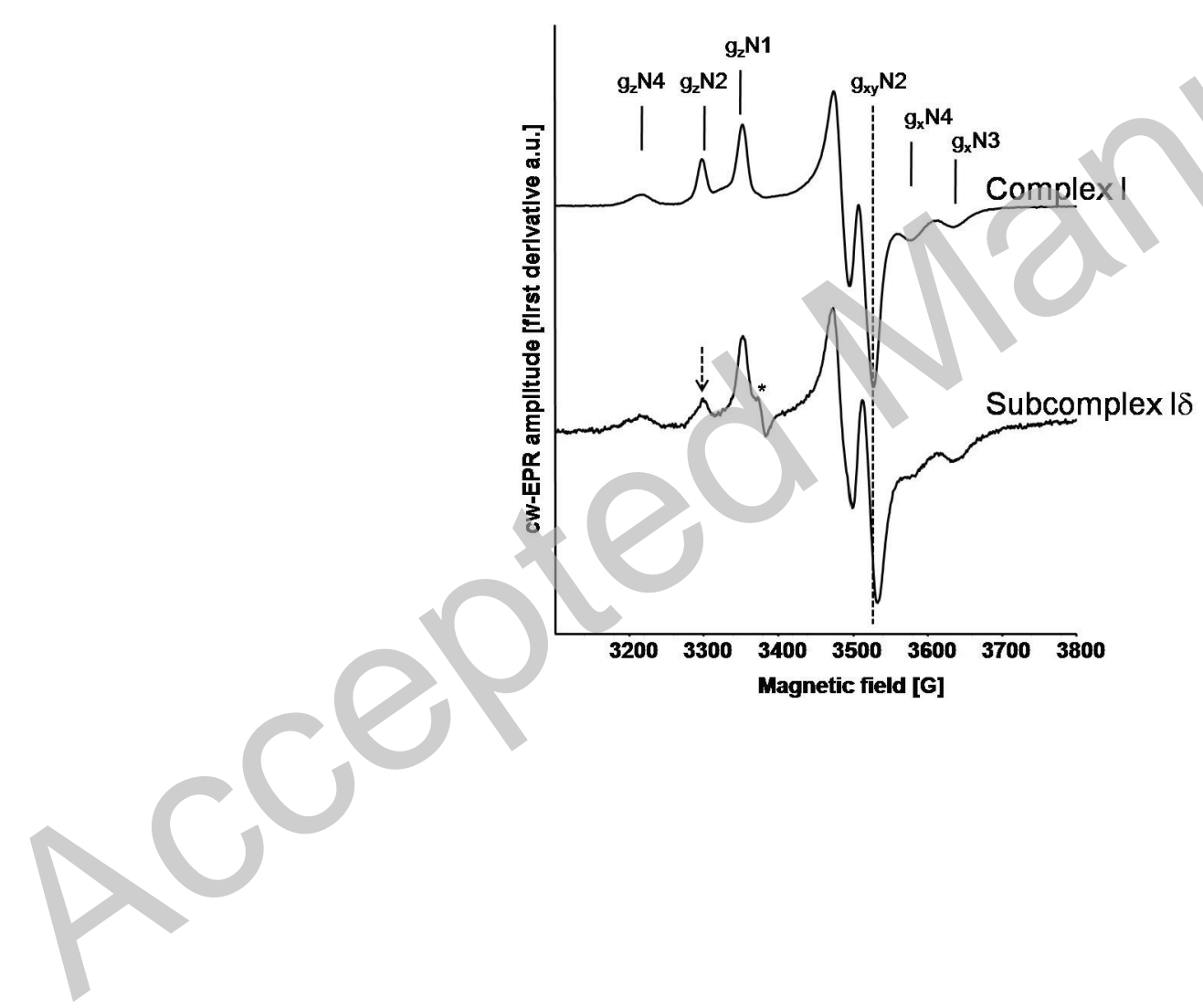




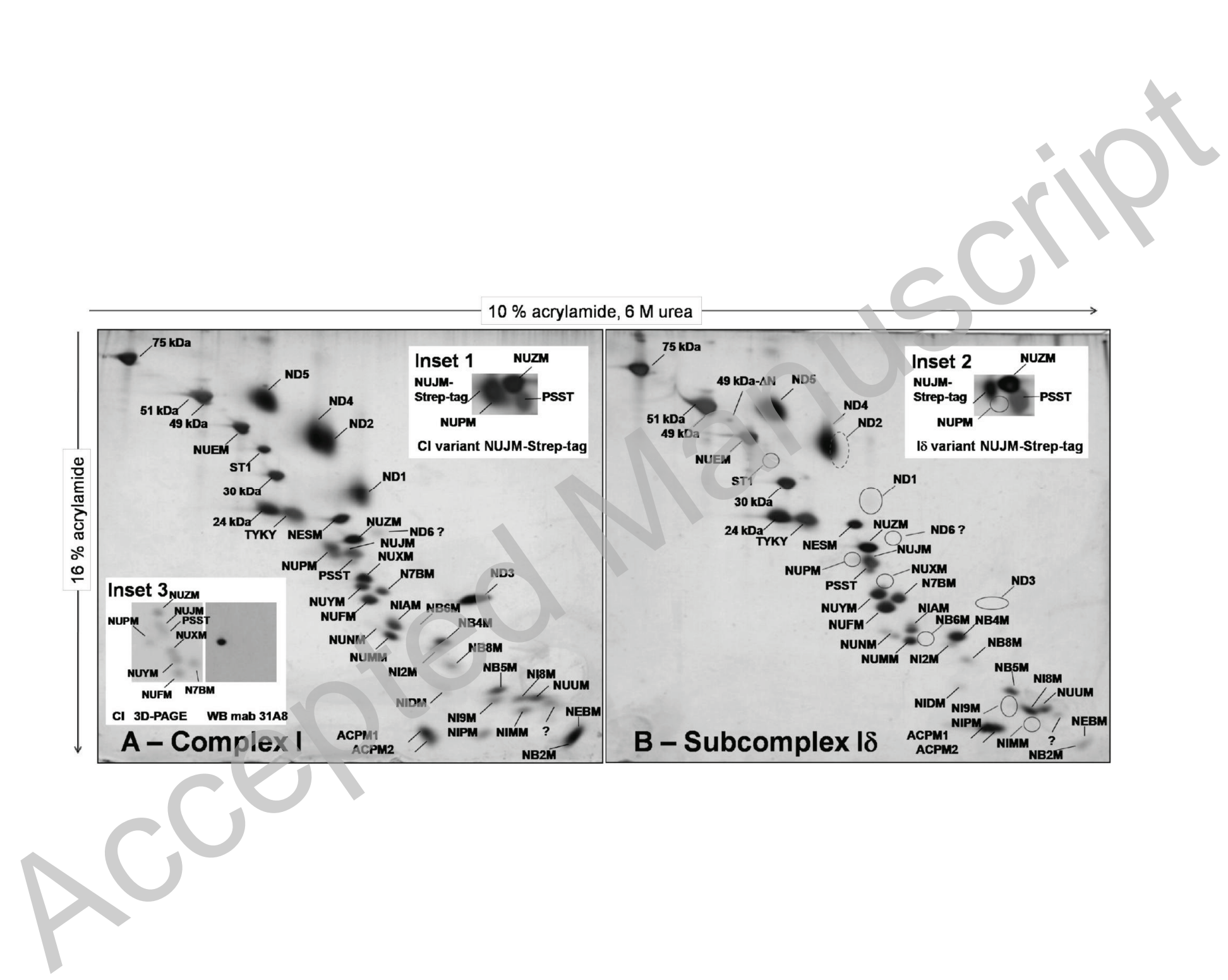




\section{B Biochemical Journal Immediate Publication. Published on 05 May 2011 as manuscript BJ20110359}

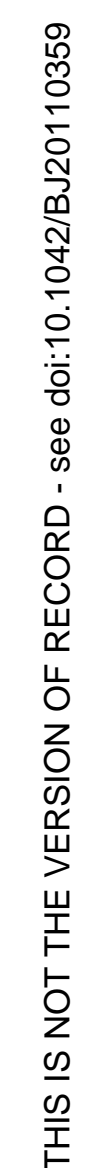

\section{Complex I NUWM-Strep-tag}

Subcomplex I8 NUWM-Strep-tag

Complex

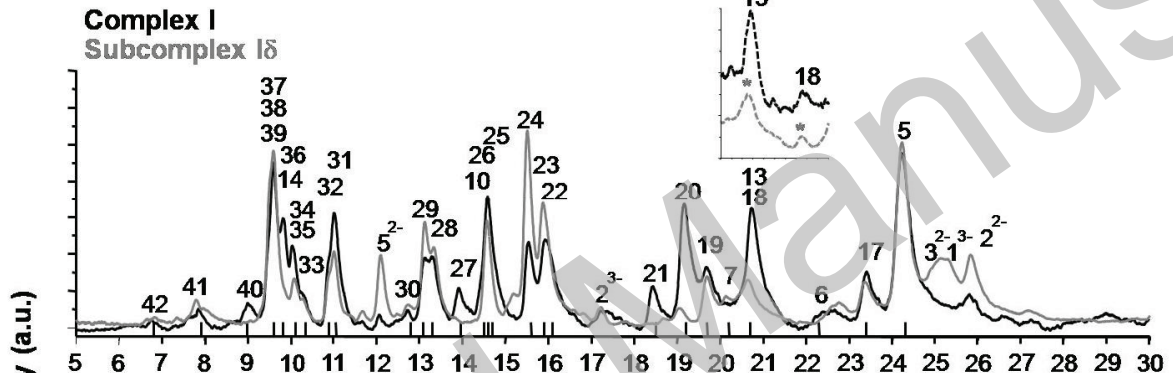

\begin{tabular}{lllllllllllllllllllllllllll}
\hline & 5 & 6 & 7 & 8 & 9 & 10 & 11 & 12 & 13 & 14 & 15 & 16 & 17 & 18 & 19 & 20 & 21 & 22 & 23 & 24 & 25 & 26 & 27 & 28 & 29 & 30
\end{tabular}

름

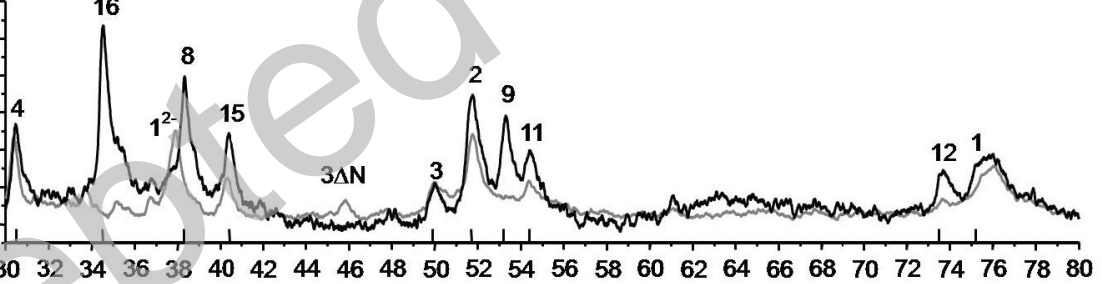

$\mathrm{m} / \mathrm{z}(\mathbf{x} 1000)$ 
B Biochemical Journal Immediate Publication. Published on 05 May 2011 as manuscript BJ20110359

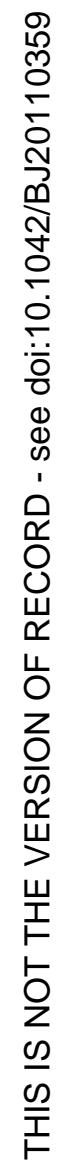

Licenced copy. Copying is not permitted, except with prior permission and as allowed by law. (C) 2011 The Authors Journal compilation (c) 2011 Portland Press Limited 


\section{B Biochemical Journal Immediate Publication. Published on 05 May 2011 as manuscript BJ20110359}

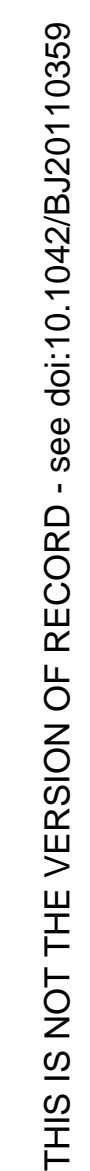

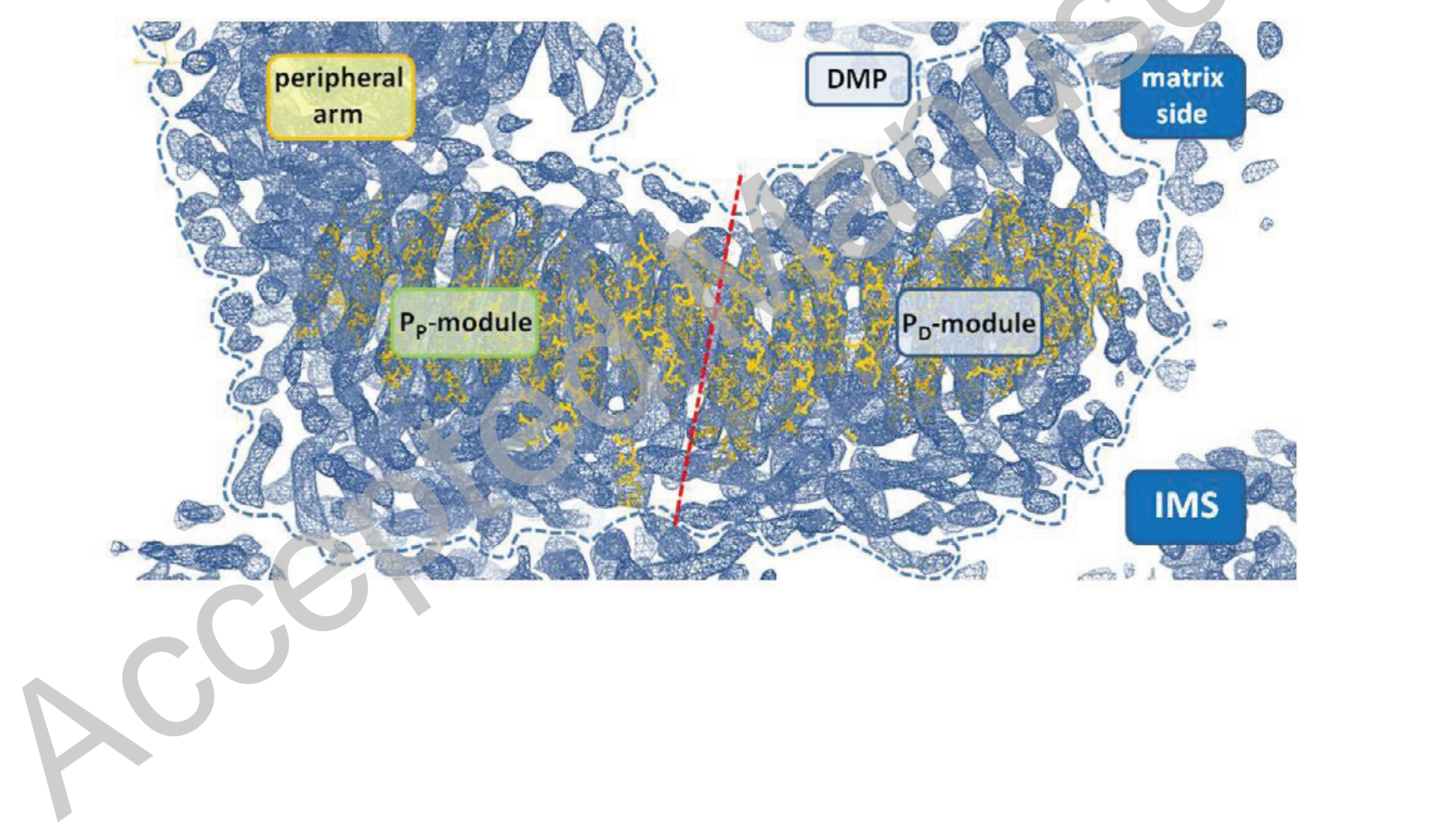

Licenced copy. Copying is not permitted, except with prior permission and as allowed by law.

(c) 2011 The Authors Journal compilation ( 22011 Portland Press Limited 